Proyecciones

Vol. 28, No 1, pp. 35-45, May 2009.

Universidad Católica del Norte

Antofagasta - Chile

\title{
ON SUMS OF BINOMIAL COEFFICIENTS
}

\author{
ANTHONY SOFO \\ VICTORIA UNIVERSITY, AUSTRALIA \\ Received: May 2008. Accepted: March 2009
}

\begin{abstract}
We investigate the integral representation of infinite sums involving the ratio of binomial coefficients. We also recover some wellknown properties of $\zeta(3)$ and extend the range of results given by other authors.
\end{abstract}

Subjclass [2000] : Primary 11B65. Secondary 05A10, 05A19, 33C20, $33 D 60$

Keywords : Triple binomial coefficients, combinatorial identities, integral representations, Zeta function. 


\section{Introduction}

In this paper we investigate the summation of the ratio of products of combinatorial coefficients. In particular, we develop integral representations for

$$
\sum_{n=0}^{\infty} \frac{t^{n} n^{p}}{\left(\begin{array}{c}
a n+j \\
j
\end{array}\right)\left(\begin{array}{c}
b n+k \\
k
\end{array}\right)\left(\begin{array}{c}
c n+l \\
l
\end{array}\right)}, \quad \sum_{n=0}^{\infty} \frac{t^{n} n^{p}\left(\begin{array}{c}
n+m-1 \\
n
\end{array}\right)}{\left(\begin{array}{c}
a n+j \\
j
\end{array}\right)\left(\begin{array}{c}
b n+k \\
k
\end{array}\right)\left(\begin{array}{c}
c n+l \\
l
\end{array}\right)}
$$

For the representation of sums of reciprocals of single and double binomial coefficients, in integral form, one may refer to some results in the papers [5], [4] and [7], see also the book [6].

For designated cases of the parameter values $(a, b, c, j, k, l, m, p, t)$, various particular sums may be expressed in terms of $\zeta(2)$ and $\zeta(3)$. For many interesting properties of the Zeta function the interested reader is refered to the internet site [9].

The representation of sums in terms of integrals is extremely useful because it allows one to estimate bounds on the sums in cases they cannot be written in closed form. Convexity properties for sums may also be investigated, see [8].

Apéry's [1], see also Beukers [2], proof of the irrationality of $\zeta(3)$ uses an elementary and quite complicated construction of the approximants $\frac{\alpha_{n}}{\beta_{n}} \in Q$ to this number based on a recurrence relation. The integral representation

$$
\int_{0}^{1} \int_{0}^{1} \int_{0}^{1} \frac{\{x(1-x) y(1-y) z(1-z)\}^{n}}{(1-(1-x y) z)^{n+1}} d x d y d z=2 \beta_{n} \zeta(3)-2 \alpha_{n}
$$

for the sequence $\left\{\alpha_{n}, \beta_{n}\right\}$ was proposed.

More recently Rhin and Viola [3] introduced the integral

$$
\int_{0}^{1} \int_{0}^{1} \int_{0}^{1} \frac{u^{h}(1-u)^{l} v^{k}(1-v)^{s} w^{j}(1-w)^{q}}{(1-(1-u v) w)^{q+h-r+1}} d u d v d w \in Q \zeta(3)+Q
$$

in their study of an irrationality measure for $\zeta(3)$.

\section{The Main Results}

In this section we develop integral identities for reciprocals of triple products of binomial coefficients.

The following lemma is given 
Lemma 1. For $a, b$ and $c$ positive real numbers and $t \in \mathbf{R}$ let

$$
f=t x^{a} y^{b} z^{c}
$$

and

$$
\lambda(f)=\sum_{n=0}^{\infty} f^{n}=\frac{1}{1-f}
$$

The consecutive partial derivative operator of the continuous function $(1-f)^{-1}$ for $(x, y, z) \in\{[0,1] \times[0,1] \times[0,1]\}$ is defined as

$$
\begin{gathered}
{[\lambda(f)]^{(0)}=\frac{1}{1-t x^{a} y^{b} z^{c}}} \\
{[\lambda(f)]^{(p)}=\underbrace{x \frac{\partial}{\partial x}\left(x \frac{\partial}{\partial x}\left(\ldots x \frac{\partial}{\partial x}\left(\frac{1}{1-f}\right)\right)\right)}_{p-\text { times }}}
\end{gathered}
$$

so that

$$
[\lambda(f)]^{(p)}=a^{p} \sum_{n=0}^{\infty} n^{p} f^{n}=\frac{a^{p}}{(1-f)^{1+p}} \sum_{r=1}^{p} S(p, r) r ! f^{r}(1-f)^{p-r},
$$

where

$$
S(p, r)=\left\{\begin{array}{l}
p \\
r
\end{array}\right\}=\frac{1}{r !} \sum_{\mu=0}^{r}(-1)^{\mu}\left(\begin{array}{c}
r \\
\mu
\end{array}\right)(r-\mu)^{p}
$$

are Stirling numbers of the second kind.

Proof. The proof follows by noting that $x \frac{\partial f}{\partial x}=$ af and

$$
\begin{aligned}
& {[\lambda(f)]^{(1)}=a \sum_{n=0}^{\infty} n f^{n}=\frac{a f}{(1-f)^{2}}} \\
& {[\lambda(f)]^{(2)}=a^{2} \sum_{n=0}^{\infty} n^{2} f^{n}=\frac{a^{2}}{(1-f)^{3}}\left\{f(1-f)+1.2 f^{2}\right\}} \\
& {[\lambda(f)]^{(3)}=a^{3} \sum_{n=0}^{\infty} n^{3} f^{n}=\frac{a^{3}}{(1-f)^{4}}\left\{f(1-f)^{2}+3.2 ! f^{2}(1-f)+3 ! f^{3}\right\}}
\end{aligned}
$$


By induction we see that $[\lambda(f)]^{(p+1)}$

$$
\begin{aligned}
& =x \frac{\partial}{\partial x}[\lambda(f)]^{(p)}=a^{p} x \frac{\partial}{\partial x}\left[\sum_{r=1}^{p} S(p, r) r ! f^{r}(1-f)^{-1-r}\right] \\
& =a^{p+1}\left[\begin{array}{c}
S(p, 1) 1 ! f(1-f)^{-2}+\ldots . .+p ! f^{p}(1-f)^{-1-p} \\
\times\{S(p, p-1)+p S(p, p)\}+(p+1) ! S(p, p) f^{p+1}(1-f)^{-2-p}
\end{array}\right] .
\end{aligned}
$$

We may write

$$
S(p, 1)=S(p+1,1)=1, S(p, p)=S(p+1, p+1)=1
$$

and by the recurrence of Stirling numbers of the second kind, $S(p+1, p)=$ $S(p, p-1)+p S(p, p)$ we have that

$$
\begin{aligned}
& a^{p+1}\left[\begin{array}{c}
S(p, 1) 1 ! f(1-f)^{-2}+\ldots .+p ! f^{p}(1-f)^{-1-p} \\
\times\{S(p, p-1)+p S(p, p)\}+(p+1) ! S(p, p) f^{p+1}(1-f)^{-2-p}
\end{array}\right] \\
= & \frac{a^{p+1}}{(1-f)^{p+2}}\left[\begin{array}{c}
S(p+1,1) 1 ! f(1-f)^{p}+\ldots . \\
S(p+1,1) p ! f^{p}(1-f)+(p+1) ! S(p+1, p+1) f^{p+1}
\end{array}\right] \\
= & \frac{a^{p+1}}{(1-f)^{p+2}} \sum_{r=1}^{p+1} S(p+1, r) r ! f^{r}(1-f)^{p+1-r},
\end{aligned}
$$

so that (2.7) follows.

Now we investigate the following theorem

Theorem 1. For $a, b$ and $c$ positive real numbers and $j, k, l \geq 0, t \in \mathbf{R}$, $p \geq 0$ then

$$
\begin{gathered}
R=\sum_{n=0}^{\infty} \frac{t^{n} n^{p}}{\left(\begin{array}{c}
a n+j \\
j
\end{array}\right)\left(\begin{array}{c}
b n+k \\
k
\end{array}\right)\left(\begin{array}{c}
c n+l \\
l
\end{array}\right)} \\
=j k l \int_{0}^{1} \int_{0}^{1} \int_{0}^{1} \frac{(1-x)^{j-1}(1-y)^{k-1}(1-z)^{l-1}}{(1-f)^{1+p}} \\
\sum_{r=1}^{p} S(p, r) r ! f^{r}(1-f)^{p-r} d x d z
\end{gathered}
$$


$\left.=T o_{j+k+l+p} F_{j+k+l+p-1}\left[\begin{array}{l}\frac{a+j}{a}, \ldots, \frac{a+1}{a}, \frac{b+k}{b}, \ldots, \frac{b+1}{b}, \frac{c+1}{b}, \frac{c+l}{c}, \ldots, \frac{c+1}{c}, 2, \ldots, 2 \\ \frac{2 a+j}{a}, \ldots, \frac{2 a+1}{a}, \frac{2 b+k}{b}, \ldots, \frac{2 b+1}{b}, \frac{2 c+1}{c} \ldots, \frac{2 c+1}{c}, 1, \ldots, 1\end{array}\right] t\right]$,

where

$$
T_{0}=\frac{t}{\left(\begin{array}{c}
a+j \\
j
\end{array}\right)\left(\begin{array}{c}
b+k \\
k
\end{array}\right)\left(\begin{array}{c}
c+l \\
l
\end{array}\right)}
$$

Proof. Consider (2.3)

$$
\begin{aligned}
R & =\sum_{n=0}^{\infty} \frac{t^{n}}{\left(\begin{array}{c}
a n+j \\
j
\end{array}\right)\left(\begin{array}{c}
b n+k \\
k
\end{array}\right)\left(\begin{array}{c}
c n+l \\
l
\end{array}\right)} \\
& =j k l \sum_{n=0}^{\infty} \frac{t^{n} \Gamma(a n+1) \Gamma(j) \Gamma(b n+1) \Gamma(k) \Gamma(c n+1) \Gamma(l)}{\Gamma(a n+j+1) \Gamma(b n+k+1) \Gamma(c n+l+1)} \\
& =j k l \sum_{n=0}^{\infty} t^{n} B(a n, j+1) B(b n, k+1) B(c n, l+1)
\end{aligned}
$$

where $\Gamma(\cdot)$ is the classical Gamma function and $B(\cdot, \cdot)$ is the Beta function.

$$
\begin{aligned}
R & =j k l \sum_{n=0}^{\infty} t^{n} \int_{x=0}^{1}(1-x)^{j-1} x^{a n} d x \int_{y=0}^{1}(1-y)^{k-1} y^{b n} d y \int_{z=0}^{1}(1-z)^{l-1} z^{c n} d z \\
& =j k l \int_{x=0}^{1} \int_{y=0}^{1} \int_{z=0}^{1}(1-x)^{j-1}(1-y)^{k-1}(1-z)^{l-1} \sum_{n=0}^{\infty}\left(t x^{a} y^{b} z^{c}\right)^{n} d x d y d z
\end{aligned}
$$

by an allowable change of integral and sum.

By Lemma 1

$$
[\lambda(f)]^{(p)}=a^{p} \sum_{n=0}^{\infty} n^{p} f^{n}=\frac{a^{p}}{(1-f)^{1+p}} \sum_{r=1}^{p} S(p, r) r ! f^{r}(1-f)^{p-r}
$$

so that 


$$
\begin{gathered}
R=j k l \int_{0}^{1} \int_{0}^{1} \int_{0}^{1} \frac{(1-x)^{j-1}(1-y)^{k-1}(1-z)^{l-1}}{(1-f)^{1+p}} \\
\sum_{r=1}^{p} S(p, r) r ! f^{r}(1-f)^{p-r} d x d z .
\end{gathered}
$$

which is the result (2.4).

The hypergeometric representation (2.5) can be obtained by the consideration of the ratio of successive terms (2.3).

We may also note that from known properties of the hypergeometric function, we may write, from (2.5):

$$
\begin{aligned}
j+k+l+p & F_{j+k+l+p-1}\left[\begin{array}{c}
\frac{a+j}{a}, \ldots, \frac{a+1}{a}, \frac{b+k}{b}, \ldots, \frac{b+1}{b}, \frac{c+l}{c}, \ldots, \frac{c+1}{c}, 2, \ldots, 2 \\
\frac{2 a+j}{a}, \ldots, \frac{2 a+1}{a}, \frac{2 b+k}{b}, \ldots, \frac{2 b+1}{b}, \frac{2 c+l}{c}, \ldots, \frac{2 c+1}{c}, 1, \ldots, 1
\end{array} \mid t\right] \\
= & a+b+c+p F_{a+b+c+p-1}\left[\begin{array}{c}
\frac{2 a}{a}, \ldots, \frac{a+1}{a}, \frac{2 b}{b}, \ldots, \frac{b+1}{b}, \frac{2 c}{c}, \ldots, \frac{c+1}{c}, 2, \ldots, 2 \\
\frac{j+2 a}{a}, \ldots, \frac{j+a+1}{a}, \frac{k+2 b}{b}, \ldots, \frac{k+b+1}{b}, \frac{l+2 c}{c}, \ldots, \frac{l+c+1}{c}, 1, \ldots, 1
\end{array} \mid t\right]
\end{aligned}
$$

\section{Example:}

1. For $a=1 / 2, b=1, c=1 / 2, j=1, k=2, l=3, p=1, t=1 / 2$

$$
\begin{aligned}
& \begin{aligned}
\mathrm{R} & =\sum_{n=0}^{\infty} \frac{2^{n}\left(\begin{array}{c}
n / 2+1 \\
1
\end{array}\right)\left(\begin{array}{c}
n+ \\
2
\end{array}\right.}{} \\
& =192 \sum_{n=0}^{\infty} \frac{n}{2^{n}(n+1)(n+2)^{3}(n+4)} \\
& =168 \zeta(3)-24\{1+4 \ln 2\} \zeta(2) \\
& =\frac{16}{315}{ }_{6} F_{5}\left[\begin{array}{c|c}
7,5,3,3,2,2 & \frac{1}{2} \\
8,6,4,4,4 & 2
\end{array}\right.
\end{aligned} \\
& =3 \int_{0}^{1} \int_{0}^{1} \int_{0}^{1} \frac{(1-y)(1-z)^{2} x^{1 / 2} y z^{1 / 2}}{\left(1-\frac{x^{1 / 2} y z^{1 / 2}}{2}\right)^{2}} d x d y d z .
\end{aligned}
$$

2. For $a=b=c=j=k=l=t=1,, p=0$, we obtain the classical result

$$
R=\sum_{n=0}^{\infty} \frac{1}{(n+1)^{3}}=\zeta(3)
$$

Now consider the following lemma and theorem, which is a generalisation of Theorem 1. 
Lemma 2. For $a, b, c$ and $m$ positive real numbers and $t \in \mathbf{R}$ let

$$
\lambda_{m}(f)=\sum_{n=0}^{\infty}\left(\begin{array}{c}
n+m-1 \\
n
\end{array}\right) f^{n}=\frac{1}{(1-f)^{m}}
$$

where $f$ is given by (2.1).

The consecutive partial derivative operator of the continuous function $(1-f)^{-m}$ for $(x, y, z) \in\{[0,1] \times[0,1] \times[0,1]\}$ is defined as

$$
\begin{aligned}
& {\left[\lambda_{m}(f)\right]^{(0)}=\frac{1}{\left(1-t x^{a} y^{b} z^{c}\right)^{m}}} \\
& \vdots \\
& {\left[\lambda_{m}(f)\right]^{(p)}=\underbrace{x \frac{\partial}{\partial x}\left(x \frac{\partial}{\partial x}\left(\ldots x \frac{\partial}{\partial x}\left(\frac{1}{1-f}\right)\right)\right)}_{p \text {-times }}}
\end{aligned}
$$

so that

$$
\begin{aligned}
& {\left[\lambda_{m}(f)\right]^{(p)}=a^{p} \sum_{n=0}^{\infty} n^{p}\left(\begin{array}{c}
n+m-1 \\
n
\end{array}\right) f^{n} } \\
= & \frac{a^{p}}{(1-f)^{m+p}} \sum_{r=1}^{p} S(p, r)(m)_{r} \quad f^{r}(1-f)^{p-r},
\end{aligned}
$$

where $S(p, r)$ are Stirling numbers of the second kind.

Proof. We note that $x \frac{\partial f}{\partial x}=a f$ and

$$
\begin{aligned}
{\left[\lambda_{m}(f)\right]^{(1)}=} & a \sum_{n=0}^{\infty} n\left(\begin{array}{c}
n+m-1 \\
n
\end{array}\right) f^{n}=\frac{m a f}{(1-f)^{m+1}} \\
{\left[\lambda_{m}(f)\right]^{(2)}=} & a^{2} \sum_{n=0}^{\infty} n^{2}\left(\begin{array}{c}
n+m-1 \\
n
\end{array}\right) f^{n}=\frac{a^{2}}{(1-f)^{m+2}}\left\{m f(1-f)+m(m+1) f^{2}\right\} \\
{\left[\lambda_{m}(f)\right]^{(3)}=} & a^{3} \sum_{n=0}^{\infty} n^{3}\left(\begin{array}{c}
n+m-1 \\
n
\end{array}\right) f^{n}=\frac{a^{3}}{(1-f)^{m+3}}\left\{m f(1-f)^{2}\right. \\
& \left.+3 . m(m+1) f^{2}(1-f)+m(m+1)(m+2) f^{3}\right\}
\end{aligned}
$$

By induction we see that

$$
\left[\lambda_{m}(f)\right]^{(p+1)}=x \frac{\partial}{\partial x}\left[\lambda_{m}(f)\right]^{(p)}=a^{p} x \frac{\partial}{\partial x}\left[\sum_{r=1}^{p} S(p, r)(m)_{r} \quad f^{r}(1-f)^{-m-r}\right]
$$




$$
=a^{p+1}\left[\begin{array}{c}
S(p, 1) m f(1-f)^{-m-1}+\ldots .+f^{p}(1-f)^{-m-p} \\
\times\left\{(m+p-1)(m)_{p-1} S(p, p-1)+p(m)_{p} S(p, p)\right\} \\
+(m+p)(m)_{p} S(p, p) f^{p+1}(1-f)^{-m-p-1}
\end{array}\right] .
$$

We may write

$$
\begin{aligned}
(m+p-1)(m)_{p-1} & =(m)_{p},(m+p)(m)_{p}=(m)_{p+1} \\
S(p, 1) & =S(p+1,1)=1, S(p, p)=S(p+1, p+1)=1
\end{aligned}
$$

and by the recurrence of Stirling numbers of the second kind, $S(p, p-1)+$ $p S(p, p)=S(p+1, p)$ we have that

$$
\begin{aligned}
a^{p+1}\left[\begin{array}{c}
S(p, 1) m f(1-f)^{-m-1}+\ldots . .+f^{p}(1-f)^{-m-p} \\
\times\left\{(m+p-1)(m)_{p-1} S(p, p-1)+p(m)_{p} S(p, p)\right\} \\
+(m+p)(m)_{p} S(p, p) f^{p+1}(1-f)^{-m-p-1}
\end{array}\right] \\
=\frac{a^{p+1}}{(1-f)^{m+p+1}}\left[\begin{array}{c}
S(p+1,1)(m)_{1} f(1-f)^{p}+\ldots . . \\
+S(p+1, p)(m)_{p} f^{p}(1-f)+(m)_{p+1} S(p+1, p+1) f^{p+1}
\end{array}\right] \\
=\frac{a^{p+1}}{(1-f)^{m+p+1}} \sum_{r=1}^{p+1} S(p+1, r)(m)_{r} f^{r}(1-f)^{p+1-r}
\end{aligned}
$$

so that (2.7) follows.

Now we investigate the following theorem

Theorem 2. For $a, b, c$ and $m$ positive real numbers $j, k, l \geq 0$ with $j+$ $k+l \geq m+p, t \in \mathbf{R}$, and $p \geq 0$ then

$$
\begin{gathered}
Q=\sum_{n=0}^{\infty} \frac{t^{n} n^{p}\left(\begin{array}{c}
n+m-1 \\
n
\end{array}\right)}{\left(\begin{array}{c}
a n+j \\
j
\end{array}\right)\left(\begin{array}{c}
b+k \\
k
\end{array}\right)\left(\begin{array}{c}
c n+l \\
l
\end{array}\right)} \\
=\sum_{n=0}^{\infty} \frac{t^{n} n^{p} j ! k ! l !(n+1)_{m-1}}{(m-1) !(a n+1)_{j}(b n+1)_{k}(c n+1)_{l}} \\
=j k l \int_{0}^{1} \int_{0}^{1} \int_{0}^{1} \frac{(1-x)^{j-1}(1-y)^{k-1}(1-z)^{l-1}}{(1-f)^{m+p}} \\
\sum_{r=1}^{p} S(p, r)(m)_{r} f^{r}(1-f)^{p-r} d x d y d z
\end{gathered}
$$


$=T_{\nabla} \quad{ }_{j+k+l+p} F_{j+k+l+p-1}$

$$
\left[\begin{array}{c}
m+1, \frac{a+j}{a}, \ldots, \frac{a+1}{a}, \frac{b+k}{b}, \ldots, \frac{b+1}{b}, \frac{c+l}{c}, \ldots, \frac{c+1}{c}, \overbrace{2, \ldots, 2}^{(p-1) \text { terms }} \\
\frac{2 a+j}{a}, \ldots, \frac{2 a+1}{a}, \frac{2 b+k}{b}, \ldots, \frac{2 b+1}{b}, \frac{2 c+l}{c}, \ldots, \frac{2 c+1}{c}, 1, \ldots, 1
\end{array} \mid t\right]
$$

where

$$
(w)_{\alpha}=w(w+1) \cdots(w+\alpha-1)=\frac{\Gamma(w+\alpha)}{\Gamma(w)}
$$

is Pochhammer's symbol, and $T_{\nabla}=m T_{0}$ where $T_{0}$ is given by (2.6).

Proof. Consider (2.8)

To arrive at the result (2.10) consider

$$
\begin{aligned}
Q= & j k l \sum_{n=0}^{\infty} t^{n}\left(\begin{array}{c}
n+m-1 \\
n
\end{array}\right) \frac{\Gamma(a n+1) \Gamma(j) \Gamma(b n+1) \Gamma(k) \Gamma(c n+1) \Gamma(l)}{\Gamma(a n+j+1) \Gamma(b n+k+1) \Gamma(c n+l+1)} \\
= & j k l \sum_{n=0}^{\infty} t^{n}\left(\begin{array}{c}
n+m-1 \\
n
\end{array}\right) B(a n+1, j) B(b n+1, k) B(c n+1, l) \\
= & j k l \sum_{n=0}^{\infty} t^{n}\left(\begin{array}{c}
n+m-1 \\
n
\end{array}\right) \int_{0}^{1}(1-x)^{j-1} x^{a n} d x \int_{0}^{1}(1-y)^{k-1} y^{b n} d y \\
& \times \int_{0}^{1}(1-z)^{l-1} z^{c n} d z \\
= & j k l \int_{0}^{1} \int_{0}^{1} \int_{0}^{1}(1-x)^{j-1}(1-y)^{k-1}(1-z)^{l-1} \sum_{n=0}^{\infty}\left(\begin{array}{c}
n+m-1 \\
n
\end{array}\right) \\
& \times\left(t x^{a} y^{b} z^{c}\right)^{n} d x d y d z
\end{aligned}
$$

by an allowable change of sum and integral.

By Lemma 2

$$
\begin{aligned}
& {\left[\lambda_{m}(f)\right]^{(p)}=a^{p} \sum_{n=0}^{\infty} n^{p}\left(\begin{array}{c}
n+m-1 \\
n
\end{array}\right) f^{n} } \\
= & \frac{a^{p}}{(1-f)^{m+p}} \sum_{r=1}^{p} S(p, r)(m)_{r} f^{r}(1-f)^{p-r},
\end{aligned}
$$

so that

$$
\begin{aligned}
S= & j k l \int_{0}^{1} \int_{0}^{1} \int_{0}^{1} \frac{(1-x)^{j-1}(1-y)^{k-1}(1-z)^{l-1}}{(1-f)^{m+p}} \\
& \sum_{r=1}^{p} S(p, r)(m)_{r} f^{r}(1-f)^{p-r} d x d y d z .
\end{aligned}
$$


which is the result (2.10).

The hypergeometric representation (2.11) can be obtained by the consideration of the ratio of successive terms (2.8) respectively.

In the case when $m=1$, Theorem 2 reduces to Theorem 1 .

Example: For $a=2, b=2, c=4, j=4, k=3, l=3, m=5, p=2, t=$ $-1$

$$
\begin{aligned}
Q= & \sum_{n=0}^{\infty} \frac{(-1)^{n} n^{2}\left(\begin{array}{c}
n+4 \\
4
\end{array}\right)}{\left(\begin{array}{c}
2 n+4 \\
4
\end{array}\right)\left(\begin{array}{c}
2 n+3 \\
3
\end{array}\right)\left(\begin{array}{l}
4 n+3 \\
3
\end{array}\right)} \\
= & 180 \int_{0}^{1} \int_{0}^{1} \int_{0}^{1} \frac{(1-x)^{3}(1-y)^{2}(1-z)^{2} x^{2} y^{2} z^{4}\left(1-5 x^{2} y^{2} z^{4}\right)}{\left(1+\frac{x^{2} y^{2} z^{4}}{2}\right)^{7}} d x d y d z \\
= & -\frac{1}{1050}{ }_{9} F_{8}\left[\begin{array}{c}
6, \frac{3}{3}, \frac{3}{2}, \frac{3}{2}, \frac{7}{4}, \frac{5}{4}, 2,2,2 \\
4, \frac{7}{2}, \frac{7}{2}, \frac{5}{2}, \frac{11}{4}, \frac{7}{4}, 3,1
\end{array} \mid 1\right] \\
= & \frac{3591}{2560}+\left(\frac{1341 \sqrt{2}}{160}-\frac{34173}{5120}\right) \pi+\frac{2223}{256} G-\frac{9}{2} \ln (2) \\
& -\frac{945}{2098} \pi \zeta(2)+\frac{2583 \sqrt{2}}{320} \ln (3-2 \sqrt{2}) .
\end{aligned}
$$

where $G$ is Catalans constant.

\section{Conclusion}

We have provided triple integral identities for sums of the reciprocal of triple binomial coefficients. In doing so we have recovered the standard representation for $\zeta(2)$ and $\zeta(3)$ and have generalised and extended some results published previously by other authors.

In another forum we shall extend our results to consider more general sums of binomial coefficients.

\section{References}

[1] R. Apéry. Irrationalitè $\zeta(2)$ and $\zeta(3)$, Journees Arithmètiques de Luminy, Ast érisque, 61, pp. 11-13, (1979).

[2] F. Beukers. A note on the irrationality of $\zeta(2)$ and $\zeta(3)$. Bull. London Math. Soc., 11 , pp. 268-272, (1979).

[3] G.Rhin and C.Viola. The group structure for $\zeta(3)$, Acta Arith. 97. 3, pp. 269-293, (2001).

[4] A. Sofo. Integral forms of sums associated with harmonic numbers, Appl. Maths. Comput., 207, pp. 356-372, (2009). 
[5] A. Sofo. General properties involving reciprocals of binomial coefficients. Journal of Integer Sequences, 9, article 06.4.5, (2006).

[6] A. Sofo. Computational Techniques for the Summation of Series. Kluwer Academic/Plenum Publishers, (2003).

[7] A. Sofo. Sums of derivatives of binomial coefficients. Adv Applied Maths, 42 (2009), pp. 123-134, (2009).

[8] A. Sofo. Convexity properties of Reciprocals of binomial coefficients. Numerical Analysis and Applied Mathematics, (2007) Editor T. E. Simos, AIP, Melville, New York, pp. 703-706.

[9] http://mathworld.wolfram.com/RiemannZetaFunction.html.

\author{
Anthony Sofo \\ School of Engineering and Science \\ Research Group in Mathematical Inequalities and Applications \\ Victoria University, \\ P. O. Box 14428 \\ Melbourne City, \\ VIC 8001, \\ Australia \\ e-mail : anthony.sofo@vu.edu.au
}

